\title{
EDITORIAL NOTE \\ Schistosoma mansoni at the Molecular and Cellular Biology of Helminth Parasites VI Meeting
}

\author{
SERGIO VERJOVSKI-ALMEIDA*
}

Molecular information about eukaryotes has increased exponentially in the last decade. Sequencing of genomes and transcriptomes of model eukaryotic organisms has been followed by efforts in many groups to obtain equivalent information on helminth parasites. Large-scale molecular information is opening a door to understanding the intricate molecular and cellular biology of complex eukaryote parasites and raising the expectations to the ability of interfering with parasite-host interactions. Recognizing the need to increase the exchange of ideas in the area of helminth parasites, the international scientific community has gathered around a specialized meeting as early as 1997, when the first "Parasitic Helminths from Genomes to Vaccines" meeting took place in Edinburgh, UK, organized by Malcolm Kennedy from the University of Glasgow, and by Mark Blaxter and Rick Maizels, from the University of Edinburgh. A second meeting took place in Edinburgh in 1999, under the same title.

In 2002 the event was renamed "Molecular and Cellular Biology of Helminth Parasites III" and it has been moved to the isle of Hydra, in Greece, where all subsequent events of the series have taken place. On September 5-10, 2010 the "Molecular and Cellular Biology of Helminth Parasites VI" meeting was organized by Kleoniki Gounaris and Murray Selkirk from the Imperial College, UK, and by Rick Maizels, from the University of Edinburgh. The meeting has an attendance limited to approximately 150 scientists, and the 2010 event comprised one keynote lecture, another 52 oral presentations with discussions in the mornings and evenings, and two poster sessions at night. We have invited several of the speakers who presented their work on Schistosoma mansoni to contribute articles to the Anais da Academia Brasileira de Ciências.

In this volume, there are five contributions on S. mansoni. All of the authors have reviewed knowledge in the specific area of their work and have contributed novel results and/or novel perspectives on their subjects of study. Evidence of non-coding RNA expression in S. mansoni is reviewed by Oliveira et al. (2011). This is an example of analysis that has only been possible because of the combined availability of the parasite genome sequence (Berriman et al. 2009) and of large scale transcriptome data which was pioneered by Franco et al. (1997) and considerably extended by Verjovski-Almeida et al. (2003). Among other topics, these authors map all publicly available ESTs to the genome sequence, thus identifying those long (> $200 \mathrm{nt}$ ) non-coding RNAs transcribed from the opposite strand of protein-coding genes and provide microarray data on the differential expression of long non-coding RNAs in different life cycle stages of the parasite (Oliveira et al. 2011).

Serpins or serine protease inhibitors are a structurally conserved family of macromolecular inhibitors found in virtually all biological systems. Quezada and McKerrow (2011) give here another very interesting example as to how the completion and annotation of the genomes of S. mansoni and S. japonicum (Zhou et al. 2009) has enabled them to identify by phylogenetic analysis two major serpin clades in schistosomes. These authors show the identification of 12 predicted serpins in these parasite genomes and they review the functional studies available in the

\footnotetext{
*Departamento de Bioquímica, Instituto de Química, Universidade de São Paulo, Av. Prof. Lineu Prestes 748, 05508-000 São Paulo, SP, Brazil.
} 
EDITORIAL NOTE

literature for schistosome and other helminths serpins. The authors raise the challenging argument that serpins may play an important role of parasite defense in a proteolytic hostile host environment, in addition to functioning in parasite homeostasis (Quezada and Mckerrow 2011).

Cyclic guanosine-3', 5'-monophosphate (cGMP)-dependent protein kinases (cGKs) belong to a conserved family of serine/threonine $(\mathrm{S} / \mathrm{T})$ kinases that in eukaryotes are modulators of cell growth, muscle tone relaxation and platelet aggregation. Here, Leutner et al. (2011) by homology screening the parasite genome identify a number of members of the class of cGKs in S. mansoni, of which one is cloned and characterized. These authors provide first evidence that SmcGK1 is expressed in the gonads of male and female worms, and that antisense messages are transcribed from the same genomic locus in the same tissues, suggesting antisense regulation of this gene. Furthermore, inhibitor studies in adult schistosomes in vitro indicate further roles of cGKs, probably associated with muscle activity (Leutner et al. 2011).

The paper by Dissous et al. (2011) reviews previously published work on S. mansoni polo-like kinase-1 within the framework that Polo-like kinases (Plks) play an important role in regulating eukaryotic cell cycle from yeast to humans. The paper revisits the role of SmPlk1 controlling gametogenesis in schistosomes using BI 2536, a specific Plk1 inhibitor. The authors compare the predicted sequences of Plk1 and Plk4 encoded in the S. mansoni genome with human Plks and confirm the subfamilies of the two SmPlks as being Plk1 and Plk4 homologs (Dissous et al. 2011). The paper reinforces the concept that parasite protein kinases might represent new targets for anti-schistosomal drugs.

Finally, Peak and Hoffmann (2011) review the quantitative methods available for screening large numbers of small molecules for their effects on a whole organism. The authors point that development of methodologies that can objectively and rapidly distinguish helminth viability or phenotype would be an invaluable tool in the anthelmintic discovery pipeline and describe how several basic techniques currently used to assess single cell eukaryote viability have been successfully applied to parasitic helminths. They additionally discuss how some of these methodologies have been adopted for high-throughput use, particularly a dual fluorescent viability assay recently developed in the authors' laboratory (Peak and Hoffmann 2011). High-throughput screening could increase the rate by which novel anthelmintics are identified and eventually translated into practical applications.

I would like to thank all the authors who have attended the meeting in Hydra and have contributed to this volume. My special thanks goes to Alexander W. A. Kellner, the journal Editor for supporting the idea of this special section.

\section{REFERENCES}

BERRIMAN M ET AL. 2009. The genome of the blood fluke Schistosoma mansoni. Nature 460: 352-358.

Dissous C, Grevelding CG AND Long T. 2011. Schistosoma mansoni Polo-like kinases and their function in control of mitosis and parasite reproduction. An Acad Bras Cienc 83: 627-635.

FRANCO GR ET AL. 1997. Evaluation of cDNA libraries from different developmental stages of Schistosoma mansoni for production of expressed sequence tags (ESTs). DNA Res 4: 231-240.

Leutner S, Beckmann S And Grevelding CG. 2011. Characterization of the cGMP-dependent protein kinase SmcGK1 of Schistosoma mansoni. An Acad Bras Cienc 83: 637-648.

Oliveira KC, Carvalho MLP, Maracaja-Coutinho V, Kitajima JP And Verjovski-Almeida S. 2011. Non-coding RNAs in schistosomes: an unexplored world. An Acad Bras Cienc 83: 673-694.

PEAK E AND HofFMANN KF. 2011. Cross-disciplinary approaches for measuring parasitic helminth viability and phenotype. An Acad Bras Cienc 83: 649-662.

Quezada LAL AND MCKerrow JH. 2011. Schistosome serine protease inhibitors: Parasite defense or homeostasis? An Acad Bras Cienc 83: 663-672.

VerJovski-AlmeidA S ET AL. 2003. Transcriptome analysis of the acoelomate human parasite Schistosoma mansoni. Nature Genetics 35: 148-157.

ZhOU Y ET AL. 2009. The Schistosoma japonicum genome reveals features of host-parasite interplay. Nature 460: $345-351$. 